\title{
Conformal Mappings in Relativistic Astrophysics
}

\author{
S. Hansraj, K. S. Govinder, and N. Mewalal \\ Astrophysics and Cosmology Research Unit, School of Mathematics, University of KwaZulu-Natal, Private Bag X54001, \\ Durban 4000, South Africa \\ Correspondence should be addressed to K. S. Govinder; govinder@ukzn.ac.za
}

Received 1 March 2013; Accepted 10 June 2013

Academic Editor: Md Sazzad Chowdhury

Copyright (C) 2013 S. Hansraj et al. This is an open access article distributed under the Creative Commons Attribution License, which permits unrestricted use, distribution, and reproduction in any medium, provided the original work is properly cited.

We describe the use of conformal mappings as a mathematical mechanism to obtain exact solutions of the Einstein field equations in general relativity. The behaviour of the spacetime geometry quantities is given under a conformal transformation, and the Einstein field equations are exhibited for a perfect fluid distribution matter configuration. The field equations are simplified and then exact static and nonstatic solutions are found. We investigate the solutions as candidates to represent realistic distributions of matter. In particular, we consider the positive definiteness of the energy density and pressure and the causality criterion, as well as the existence of a vanishing pressure hypersurface to mark the boundary of the astrophysical fluid.

\section{Introduction}

The gravitational evolution of celestial bodies may be modeled by the Einstein field equations. These are a system of ten highly coupled partial differential equations expressing an equivalence between matter and geometry. The equations are extremely difficult to solve in general and so simpler cases have to be treated to gain an understanding into how certain types of matter behave under the influence of the gravitational field. For example, the most studied configuration of a matter distribution is that of a static spherically symmetric perfect fluid. The assumption of spherical symmetry has the effect of reducing the field equations to a system of three equations in four unknowns if the matter is neutral. While this is a severe, but reasonable, restriction, even in this case not all solutions to the system of field equations have been found. Work on this problem has been ongoing since the first exact solution appeared in 1916 when Karl Schwarzschild published his solution for a vacuum (matter free), and, to the present, this exterior solution continues to be used to model phenomena such as black holes. This solution is unique, and, moreover, Birkhoff [1] showed that the solution is independent of whether the sphere is static or not. In other words, the Schwarzschild exterior solution [2] is simply a consequence of the spherical geometry. From this theorem also follows the conclusion that pulsating fluid spheres do not generate gravitational waves.
By considering the case of a uniform density sphere, Schwarzschild [3] found a unique interior solution. However, the problem of finding all possible solutions for a nonconstant energy density is still an open problem. Since the system of field equations is underdetermined, one of the geometric or dynamical variables must be specified at the outset. This freedom of choice renders it impractical to determine all possible solutions. A comprehensive review of the static spherically symmetric fluid sphere has been compiled by Delgaty and Lake [4]. Recent work by Fodor [5], Martin and Visser [6], Lake [7], Boonserm et al. [8], and Rahman and Visser [9], which resuscitated an idea first proposed by Wyman [10], reported algorithms for finding all possible exact solutions. However, each prescription required an integration or two which may be intractable in practice given that a certain variable had to be selected in some ad hoc fortuitous way. In any event, even if solutions to the field equations are found, they have to satisfy certain physical requirements to be considered candidates for realistic matter. Finch and Skea [11] studied over 100 exact solutions and have found that only about 16 satisfy the most elementary physical requirements. It should be remarked that the importance of some of the so called requirements for physical plausibility is debatable given that the gravitational processes inside a star may not be accurately determined.

If spherical symmetry is maintained and if, in addition, the matter distribution contained charge, then the Einstein 
field equations must be supplemented by Maxwell's equations which incorporate the effects of the electromagnetic field. These Einstein-Maxwell equations constitute a set of six field equations in four unknowns. In this case, the problem is simpler as now two of the matter or geometrical quantities must be chosen at the beginning and the remaining four will follow from the integration of the field equations. A detailed collection of the two-variable choices that have been considered has been achieved by Ivanov [12]. The caveat is that, although there is an extra degree of freedom, finding physically palatable solutions is extremely rare. Additionally, it is required that the exact solution for the interior be matched with the unique exterior solution for charged spheres according to Reissner [13] and Nordstrom [14]. The solution of Hansraj and Maharaj [15] in the form of Bessel functions of half integer order was shown to satisfy elementary physical requirements. Other matter configurations include radiation and rotation. In each case a unique exterior solution has been found-the exterior metric for a radiating star is credited to Vaidya [16], while an exterior metric for a rotating sphere was constructed by Kerr [17]. While in the case of radiating spheres many interior solutions have been found, this is not the case for a rotating sphere. The problem of finding a solution to the Einstein field equations that incorporate rotations is still unsolved problem in classical general relativity.

The mathematical approach described earlier often relies on choosing functional forms for some of the variables that eventually allow for the integration of the entire system of differential equations. Fortunately, the analysis is simplified by the fact that a single master equation holds the key to unlocking the whole system. This master equation may be interpreted as a second-order linear differential equation (see Duorah and Ray [18], Durgapal and Bannerji [19], and Finch and Skea [20]) or as a first-order Ricatti equation (for example, see Lake [7] and Fodor [5]). Once a solution is found, then the physical and geometric quantities must be established and checked for physical plausibility. An alternative approach is to impose some physical constraints $a$ priori, for example, to prescribe an equation of state relating the pressure and energy density. However, the drawback of this approach is that the field equations may not be solvable. Only a few solutions of this kind have been reported in the literature. Interestingly, the charged analogue of the FinchSkea [20] stars reported by Hansraj and Maharaj [15] turned out to possess a barotropic equation of state. This is a desirable feature of physically reasonable perfect fluids.

The situation when considering nonstatic (that is, time dependent) matter configurations introduces another level of complexity into the problem. The partial differential equations are now in terms of the spatial and temporal coordinates. Finding exact solutions usually amounts to prescribing relationships between the geometric quantities in order to solve the field equations completely. Therefore, in view of this difficulty, we consider an alternative mathematical approach. We employ conformal mappings on existing (possibly defective) solutions possessing Killing algebras (see Table 1) with the intention of solving the now conformally related Einstein field equations. The reason for the potential success of this
TABLE 1

\begin{tabular}{lc}
\hline (1) $\psi=0$ & $\mathbf{X}$ is a Killing vector \\
(2) $\psi_{; a}=0 \neq \psi$ & $\mathbf{X}$ is a homothetic Killing vector \\
(3) $\psi_{; a b}=0 \neq \psi_{; a}$ & $\mathbf{X}$ is a special conformal Killing vector \\
(4) $\psi_{; a b} \neq 0$ & $\mathbf{X}$ is a nonspecial conformal Killing vector \\
\hline
\end{tabular}

approach has to do with the fact that the existence of conformal Killing vectors is known to simplify the field equationsin other words, they involve a geometric constraint. This is in opposition to an algebraic constraint which may be imposed, for example, by demanding that the eigenvectors of the Weyl tensor have certain preferred alignments. This gives rise to the Petrov [21] classification scheme which in reality is a result in pure mathematics applicable to any Lorentzian manifold. In our approach, the procedure is aided by the Defrise-Carter [22] theorem which specifies how conformal Killing vectors become Killing vectors under conformal transformations. The theorem states the following: suppose that a manifold $(M, \mathbf{g})$ is neither conformally flat nor conformally related to a generalised plane wave. Then, a Lie algebra of conformal Killing vectors on $M$ with respect to $\mathbf{g}$ can be regarded as a Lie algebra of Killing vectors with regard to some metric on $M$ conformally related to $\mathbf{g}$ [23].

The benefit of utilising this approach is that we may utilise a known exact solution of the Einstein field equations and then solve the associated conformally related equations. This is because the conformal Einstein tensor splits neatly into the original Einstein tensor and a conformally related part. So, for example, if one begins with a vacuum seed solution, then it is known that the Einstein tensor is zero and so only the conformal part must now be considered in conjunction with a perfect fluid energy momentum tensor. Such solutions are referred to as conformally Ricci-flat spacetimes. This scheme has yielded useful results. A pioneering work on this method was conducted by van den Bergh [24, 25], and, recently Castejón-Amenedo and Coley [26] and Hansraj et al. [27] found new classes of exact solutions that were nonstatic. In fact, the fluid congruences were found to be accelerating, shearing, and expanding, which is a category of solution types that is rare. Hansraj [28] analysed the conjecture of van den Bergh [24] that perfect fluid spacetimes can be found by using the nonconformally flat Schwarzschild exterior solution. It was proved that all such spacetimes are necessarily static, and a class of exact solutions were reported. In Hansraj et al. [27], use was made of a nonvacuum seed solution however, no physical analysis of the resulting solutions was carried out. Govinder and Hansraj [29] completely analysed the most general set of field equations with the help of Lie group analysis methods and found new exact solutions. As the solutions were four dimensional, it was not possible to provide a full physical analysis. We consider these aspects in the current paper.

\section{Differential Geometry}

A brief consideration of the mathematical framework for this problem will be in order. We consider a spacetime $(M, g)$, 
where $M$ is a 4-dimensional differentiable manifold with respect to a symmetric, nonsingular metric field $g$. Points in $M$ are labelled by real coordinates, three of which are spacelike and one is timelike. The points are represented as $\left(x^{a}\right)=$ $\left(x^{0}, x^{1}, x^{2}, x^{3}\right)$, where $x^{0}$ is timelike and $x^{1}, x^{2}$, and $x^{3}$ are spacelike.

The invariant distance between neighbouring points of a curve in $M$ is defined by the fundamental metric form

$$
d s^{2}=g_{a b} d x^{a} d x^{b},
$$

where $g_{a b}$ is referred to as the metric tensor. It is symmetric and possesses an inverse $g^{a b}=1 / g_{a b}$. The Christoffel symbol $\Gamma$ is a metric connection that preserves inner products under parallel transport. The coefficients of $\Gamma$ are calculated by

$$
\Gamma_{b c}^{a}=\frac{1}{2} g^{a d}\left(g_{c d, b}+g_{d b, c}-g_{b c, d}\right),
$$

where commas denote partial differentiation.

The Riemann curvature tensor (also known as RiemannChristoffel tensor) is a $(1,3)$ tensor field whose coordinate components are given in terms of the coordinate components of the connection as follows:

$$
R_{b c d}^{a}=\Gamma_{b d, c}^{a}-\Gamma_{b c, d}^{a}+\Gamma_{e c}^{a} \Gamma_{b d}^{e}-\Gamma_{e d}^{a} \Gamma_{b c}^{e} .
$$

The Ricci tensor $R_{a b}$, obtained by contraction of the Riemann curvature tensor, is given by

$$
R_{a b}=\Gamma_{a b, d}^{d}-\Gamma_{a d, b}^{d}+\Gamma_{a b}^{e} \Gamma_{e d}^{d}-\Gamma_{a d}^{e} \Gamma_{e b}^{d} .
$$

Upon contraction of the Ricci tensor, we obtain the Ricci scalar $R$, given by

$$
R=g^{a b} R_{a b} .
$$

The Einstein tensor $G_{a b}$ is obtained from the Ricci tensor and the Ricci scalar in the following way:

$$
G_{a b}=R_{a b}-\frac{1}{2} R g_{a b} .
$$

For matter in its neutral state, the energy-momentum tensor $T_{a b}$ is given by

$$
T_{a b}=(\mu+p) u_{a} u_{b}+p g_{a b}+q_{a} u_{b}+q_{b} u_{a}+\pi_{a b},
$$

where $\mu$ is the energy density, $p$ is the isotropic pressure, $q_{a}$ is the heat flow vector, $\pi_{a b}$ is the stress tensor and $u_{a}$ is the velocity field vector.

For a perfect fluid, the energy-momentum tensor, reduces to

$$
T_{a b}=(\mu+p) u_{a} u_{b}+p g_{a b} .
$$

The energy-momentum tensor (8) and the Einstein tensor (6) are related via the Einstein field equations in the following way:

$$
G_{a b}=T_{a b} .
$$

This is a system of 10 partial differential equations which are highly nonlinear and hence difficult to integrate in general.

It is also of importance to our work to mention the Weyl conformal tensor which has physical relevance in expressing the tidal force a body experiences when moving along a geodesic. In fact, the Weyl tensor is the traceless component of the Riemann tensor and is given by

$$
\begin{aligned}
C_{a b c d}= & R_{a b c d}+\frac{1}{(n-1)(n-2)} R\left(g_{a c} g_{b d}-g_{a d} g_{b c}\right) \\
& -\frac{1}{n-2}\left(g_{a c} R_{b d}-g_{b c} R_{a d}+g_{b d} R_{a c}-g_{a d} R_{b c}\right) .
\end{aligned}
$$

\section{Conformal Geometry}

A variety of symmetries may be defined on the manifold by the action of $\mathscr{L}_{\mathrm{X}}$ on the metric tensor and associated quantities $[30,31]$. Of the various symmetries that are possible, we are primarily concerned with conformal motions. A conformal Killing vector $\mathbf{X}$ is defined by the action of $\mathscr{L}_{\mathbf{X}}$ on the metric tensor field $\mathbf{g}$ so that

$$
\mathscr{L}_{\mathbf{x}} g_{a b}=2 \psi g_{a b},
$$

where $\psi\left(x^{a}\right)$ is the conformal factor. There are four special cases associated with (11), namely.

The spanning set $\left\{\mathbf{X}_{A}\right\}=\left\{\mathbf{X}_{1}, \mathbf{X}_{2}, \ldots, \mathbf{X}_{r}\right\}$ of all the conformal Killing vectors of a spacetime generates a Lie algebra. The elements of this basis are related by

$$
\begin{aligned}
{\left[\mathbf{X}_{A}, \mathbf{X}_{B}\right] } & =\mathbf{X}_{A} \mathbf{X}_{B}-\mathbf{X}_{B} \mathbf{X}_{A} \\
& =C^{D}{ }_{A B} \mathbf{X}_{D},
\end{aligned}
$$

where the quantities $C^{D}{ }_{A B}$ are the structure constants of the group. The structure constants have the property of being independent of the coordinate system but do depend on the choice of the basis. The structure constants are skew symmetric so that

$$
C^{D}{ }_{A B}=-C_{B A}^{D}
$$

and satisfy the identity

$$
C^{E}{ }_{A D} C^{D}{ }_{B C}+C^{E}{ }_{B D} C^{D}{ }_{C A}+C^{E}{ }_{C D} C^{D}{ }_{A B}=0 .
$$

The integrability condition for the existence of a conformal symmetry is given by

$$
\mathscr{L}_{\mathbf{X}} C^{a}{ }_{b c d}=0 .
$$

Suppose that we are given a spacetime $(M, \mathbf{g})$ with line element

$$
d s^{2}=g_{a b} d x^{a} d x^{b}
$$

and a related spacetime $(M, \overline{\mathbf{g}})$ with the line element

$$
d \bar{s}^{2}=\bar{g}_{a b} d x^{a} d x^{b} .
$$


Then, the previous two line elements are said to be conformally related if

$$
\bar{g}_{a b}=e^{2 U} g_{a b}, \quad \bar{g}^{a b}=e^{-2 U} g^{a b},
$$

where $U\left(x^{c}\right)$ is a nonzero, real-valued function of the coordinates on $M$. This transformation between $\overline{\mathbf{g}}$ and $\mathbf{g}$ is called a conformal transformation and is a special type of mapping between metric spaces given by dilatation (or contraction) of all lengths by a common factor which varies from point to point. If $\mathbf{X}$ is a conformal Killing vector in the $(M, \mathbf{g})$ spacetime so that (11) holds, that is,

$$
\mathscr{L}_{\mathbf{X}} g_{a b}=2 \psi g_{a b},
$$

then $\mathbf{X}$ is also a conformal Killing vector in the related spacetime $(M, \overline{\mathbf{g}})$, and we have

$$
\mathscr{L}_{\mathbf{x}} \bar{g}_{a b}=2 \sigma \bar{g}_{a b} .
$$

With the help of (11), (18), and (20), we can show that the factors $\sigma$ and $\psi$ are related by

$$
\sigma=\left[\frac{\mathscr{L}_{\mathbf{X}}\left(e^{2 U}\right)}{2 e^{2 U}}+\psi\right] .
$$

From this, we can conclude that conformal transformations map conformal Killing vectors to conformal Killing vectors, with the conformal factors being different. However, conformal transformations do not map Killing vectors or homothetic Killing vectors to their respective counterparts in the conformally related spacetime. Note that, in the trivial case where $e^{2 U}$ is constant, we have $\sigma=\psi$. However, it is important to note that the converse does not hold. If $\sigma=\psi$, then (21) implies that $\mathscr{L}_{\mathbf{X}}\left(e^{2 U}\right)=0$. This means that $U$ is constant along the integral curves of the vector $\mathbf{X}$, but it may vary elsewhere on the manifold.

It should be noted that the connection coefficients, Riemann curvature tensor, Ricci tensor, and Ricci scalar for the metric $g_{a b}$ are related to those of the metric $\bar{g}_{a b}=e^{2 U} g_{a b}$ by the following formulae which are given in de Felice and Clarke [32]:

$$
\begin{aligned}
\bar{\Gamma}_{b c}^{a}= & \Gamma_{b c}^{a}+\frac{1}{2}\left[\delta_{b}^{a} \phi_{c}+\delta_{c}^{a} \phi_{b}-g_{b c} \phi^{a}\right], \\
\bar{R}_{b c d}^{a}= & R_{b c d}^{a}+\delta_{[d}^{a} \nabla_{c]} \phi_{b}+g_{b[c} \nabla_{d]} \phi^{a} \\
& +\frac{1}{2} \delta^{a}{ }_{[c} \phi_{d]} \phi_{b}-\frac{1}{2} g_{b[c} \phi_{d]} \phi^{a} \\
& -\frac{1}{2} \delta^{a}{ }_{[c} g_{d] b} \phi^{e} \phi_{e}, \\
\bar{R}_{b d}= & R_{b d}-\frac{1}{2}\left[2 \phi_{b ; d}-\phi_{a} \phi_{b}+g_{a b} \phi^{e} \phi_{e}\right] \\
& -\frac{1}{2} g_{b d} \phi_{; a}^{a}, \\
\bar{R}= & \frac{1}{\Omega}\left[R-3 \phi_{; e}^{e}-\frac{3}{2} \phi_{e} \phi^{e}\right],
\end{aligned}
$$

where we have defined $\phi_{a}=\partial_{a}(\ln \Omega)$ and $\Omega=e^{2 U}$. Additionally, the conformal Einstein tensor $\overline{\mathbf{G}}$ is given by

$$
\begin{aligned}
\bar{G}_{a b}= & G_{a b}+2\left(U_{a} U_{b}-\frac{1}{2} U^{c} U_{c} g_{a b}\right) \\
& +2\left(U^{c}{ }_{i c}+U^{c} U_{c}\right) g_{a b}-2 U_{a ; b},
\end{aligned}
$$

where the covariant derivatives and contractions are calculated on the original metric $g_{a b}$. Note that we may also write the conformal Einstein tensor (23) as

$$
\begin{aligned}
\bar{G}_{a b}= & G_{a b}-2 U_{a} U_{b}-2 U_{a ; b} \\
& +\left(2 U_{; c}^{c}-U^{c} U_{c}\right) \bar{g}_{a b},
\end{aligned}
$$

where the geometric quantities are now evaluated using the conformally related metric $\bar{g}_{a b}[33]$.

An important characteristic of conformal mappings is that the Weyl tensor $C$ is invariant under the transformation; that is,

$$
\bar{C}_{a b c d}=C_{a b c d},
$$

and consequently a conformal transformation is sometimes referred to as a Weyl rescaling in the literature. A necessary and sufficient condition that a spacetime is conformally flat is that the Weyl tensor $\mathbf{C}$ vanishes.

The covariant derivative of the timelike fluid 4-vector field $\mathbf{u}$ can be decomposed as follows

$$
u_{a ; b}=\sigma_{a b}+\frac{1}{3} \Theta h_{a b}-\dot{u}_{a} u_{b}+\omega_{a b},
$$

where $h_{a b}=g_{a b}+u_{a} u_{b}$ is the projection tensor. In the previous, we have defined

$$
\begin{gathered}
\dot{u}_{a}=u_{a ; b} u^{b}, \\
\omega_{a b}=u_{[a ; b]}+\dot{u}_{[a} u_{b]}, \\
\sigma_{a b}=u_{(a ; b)}+\dot{u}_{(a} u_{b)}-\frac{1}{3} \Theta h_{a b}, \\
\Theta=u_{; a}^{a},
\end{gathered}
$$

where $\dot{u}_{a}$ is the acceleration vector $\left(\dot{u}^{a} u_{a}=0\right), \omega_{a b}$ is the skew-symmetric vorticity tensor $\left(\omega_{a b} u^{a}=0\right), \sigma_{a b}$ is the symmetric shear tensor $\left(\sigma_{a b} u^{b}=0=\sigma_{a}^{a}\right)$, and $\Theta$ is the rate of expansion.

Under a conformal transformation $\bar{g}_{a b}=e^{2 U} g_{a b}$, the world lines are the same and the velocity field transforms as

$$
\bar{u}_{a}=e^{U} u_{a}
$$

and we obtain

$$
\begin{gathered}
\overline{\dot{u}}_{a}=e^{U}\left(\dot{u}_{a}+u_{a} u_{b} U^{, b}+U_{, a}\right), \\
\bar{\Theta}=e^{-U} \Theta-3 u^{a}\left(e^{-U}\right)_{, a}, \\
\bar{\omega}_{a b}=e^{U} \omega_{a b}, \\
\bar{\sigma}_{a b}=e^{U} \sigma_{a b}
\end{gathered}
$$


for the transformed kinematical quantities listed in (27). The quantities, (29) will be useful in studying the physical behaviour of the models generated by a conformal transformation [27].

\section{Einstein Field Equations}

We consider the metric

$$
d s^{2}=-d t^{2}+d x^{2}+e^{2 v(y, z)}\left(d y^{2}+d z^{2}\right)
$$

which is of Petrov type D. Solving (11) for this metric reveals that (30) admits three Killing vectors, namely,

$$
\begin{gathered}
X_{1}=\partial_{t}, \\
X_{2}=\partial_{x}, \\
X_{3}=x \partial_{t}+t \partial_{x}
\end{gathered}
$$

with the Lie bracket relations

$$
\begin{aligned}
& {\left[X_{1}, X_{2}\right]=0,} \\
& {\left[X_{1}, X_{3}\right]=X_{2} \text {, }} \\
& {\left[X_{2}, X_{3}\right]=X_{1} \text {. }}
\end{aligned}
$$

The conformally related analogue is given by

$$
d s^{2}=e^{2 U(t, x, y, z)}\left[-d t^{2}+d x^{2}+e^{2 v(y, z)}\left(d y^{2}+d z^{2}\right)\right] .
$$

By the Defrise-Carter [22] theorem, the previous Killing vectors are now conformal Killing vectors, given by

$$
\begin{gathered}
Y_{1}=U_{t}, \\
Y_{2}=U_{x}, \\
Y_{3}=x U_{t}+t U_{x} .
\end{gathered}
$$

To determine the perfect fluid energy-momentum tensor, we select a fluid 4 -velocity vector $\mathbf{u}$ that is noncomoving of the form

$$
u^{a}=e^{-U}\left(\cosh v \delta_{0}^{a}+\sinh v \delta_{1}^{a}\right),
$$

where $v=v(t, x)$. Note that utilising a comoving velocity field would also lead to conformal flatness, and this case is not of immense interest as explained previously.
The coupling of the conformal Einstein tensor and the transformed energy momentum tensor yields the Einstein field equations given by

$$
\begin{gathered}
U_{t} U_{y}-U_{t y}=0, \\
U_{t} U_{z}-U_{t z}=0, \\
U_{x} U_{y}-U_{x y}=0, \\
U_{x} U_{z}-U_{x z}=0, \\
U_{t} U_{x}-U_{t x}=-\frac{1}{4}(\mu+p) e^{2 U} \sinh 2 v, \\
U_{y} U_{z}-U_{y z}+v_{z} U_{y}+v_{y} U_{z}=0, \\
-2 U_{x x}-U_{x}^{2}+3 U_{t}^{2} \\
-e^{-2 v}\left(2 U_{y y}+2 U_{z z}+U_{y}^{2}+U_{z}^{2}+v_{y y}+v_{z z}\right) \\
=(\mu+p) e^{2 U} \cosh ^{2} v-p e^{2 U}, \\
-2 U_{t t}-U_{t}^{2}+3 U_{x}^{2} \\
+e^{-2 v}\left(2 U_{y y}+2 U_{z z}+U_{y}^{2}+U_{z}^{2}+v_{y y}+v_{z z}\right) \\
=(\mu+p) e^{2 U} \sinh ^{2} v+p e^{2 U}, \\
2 U_{z z}+U_{z}^{2}+3 U_{y}^{2}+2 v_{y} U_{y}-2 v_{z} U_{z} \\
+e^{2 v}\left(2 U_{x x}-2 U_{t t}+U_{x}^{2}-U_{t}^{2}\right)=p e^{2 v+2 U}, \\
2 U_{y y}+U_{y}^{2}+3 U_{z}^{2}-2 v_{y} U_{y}+2 v_{z} U_{z} \\
+e^{2 v}\left(2 U_{x x}-2 U_{t t}+U_{x}^{2}-U_{t}^{2}\right)=p e^{2 v+2 U}
\end{gathered}
$$

for the line element (33).

The field equations may be reduced to a simpler form by observing that an immediate consequence of (36)-(39) is the functional form

$$
e^{-U}=f(t, x)+h(y, z),
$$

where $f$ and $h$ are arbitrary functions and $U=U(t, x, y, z)$. After a series of arduous calculations, it may be shown that (36)-(45) reduce to the simpler set of equations

$$
\begin{aligned}
& \mu=3\left(f_{t}^{2}-f_{x}^{2}\right)+(f+h)(4 k f-2 k h+3 \alpha) \\
&-3 e^{-2 v}\left(h_{y}^{2}+h_{z}^{2}\right), \\
& p=-3\left(f_{t}^{2}-f_{x}^{2}\right)+(f+h)\left(2 f_{t t}-2 f_{x x}+2 k h-\alpha\right)+3 e^{-2 v}\left(h_{y}^{2}+h_{z}^{2}\right), \\
& \tanh ^{2} v=\frac{2 f_{x x}-2 k f-\alpha}{2 f_{t t}+2 k f+\alpha}, \\
& f_{t x}^{2}=\frac{1}{4}\left(2 f_{x x}-2 k f-\alpha\right)\left(2 f_{t t}+2 k f+\alpha\right),
\end{aligned}
$$




$$
\begin{aligned}
& h_{y z}=v_{z} h_{y}+v_{y} h_{z}, \\
& h_{y y}-h_{z z}=2 v_{y} h_{y}+2 v_{z} h_{z},
\end{aligned}
$$

where $\alpha$ is a separation constant and we have set $\nu_{y y}+\nu_{z z}=$ $-2 k e^{2 v}, k$ being a constant. Note that the expression $v_{y y}+v_{z z}$ is nonzero. If this expression was zero, the Weyl tensor would vanish leading to conformally flat solutions. Such solutions have already been fully determined as Stephani [34] stars in the case of expansion or generalised Schwarzschild metrics for no expansion. The set of (47)-(52) has been discussed in general by Govinder and Hansraj [29] with the help of the Lie group analysis approach. New exact solutions were reported; however, the difficulty of working with such solutions is that they are 4 dimensional and therefore it is not transparent whether the solutions could represent physically acceptable matter configurations. For this reason, we elect to study some physical properties by neglecting the $y$ and $z$ directional contributions-that is, we set $h=0$ ala Castejón-Amenedo and Coley [26].

\section{The Static Case}

As a first step, it is of interest to analyse the behaviour of our model when it is time independent. That is, we consider the conformal factor in the form $U=U(x)$. The system of field equations (47)-(52) reduces to

$$
\begin{gathered}
\mu=-3 f_{x}^{2}+f(4 k f+3 \alpha), \\
p=3 f_{x}^{2}-f\left(2 f_{x x}+\alpha\right), \\
\tanh ^{2} v=\frac{2 f_{x x}-2 k f-\alpha}{2 k f+\alpha}, \\
0=\left(2 f_{x x}-2 k f-\alpha\right)(2 k f+\alpha),
\end{gathered}
$$

where it should be recalled that $\alpha$ is a separation constant (effectively an integration constant) and $k$ is an arbitrary constant. Equation (56) leads to $f=-\alpha / 2 k$ which in turn implies a constant conformal factor. This is trivial. However, another consequence of (56) is the equation

$$
2 f_{x x}-2 k f-\alpha=0 .
$$

Equation (57) is a standard second-order differential equation with constant coefficients and its solution may be categorised as follows (note in what follows that we take the coupling constant $\alpha$ to be positive without loss of generality:

$$
f(x)= \begin{cases}\frac{\alpha}{4} x^{2}+C_{1} x+C_{2}, & k=0, \\ -\frac{\alpha}{2 k}+C_{1} \cosh \sqrt{k} x+C_{2} \sinh \sqrt{k} x, & k>0, \\ \frac{\alpha}{2 k}+C_{1} \cos \sqrt{-k} x+C_{2} \sin \sqrt{-k} x, & k<0,\end{cases}
$$

where $C_{1}$ and $C_{2}$ are integration constants.

5.1. The Case $k=0$. This case is not particularly interesting because $k=0$ results in a metric that is conformally flat.
Such metrics have been found up to integration in general. However, in this case, we are able to provide the explicit forms for the kinematic and dynamical variables. When $k=0$, the complete solution of the conformally related Einstein field equations is given by

$$
\begin{aligned}
& e^{-U(x)}=\frac{\alpha}{4} x^{2}+C_{1} x+C_{2}, \\
& \begin{aligned}
& \rho= \frac{1}{4} k \alpha^{2} x^{4}+2 k \alpha C_{1} x^{3}+2 k\left(\alpha C_{2}+2 C_{1}^{1}\right) x^{2} \\
& \quad+8 k C_{1} C_{2} x+\left(4 k C_{2}^{2}+3 C_{2}-3 C_{1}^{2}\right), \\
& p= \frac{1}{4} \alpha^{2} x^{2}-\alpha C_{1} x+\left(3 C_{1}^{2}-2 \alpha C_{2}\right), \\
& \tanh v=0 .
\end{aligned}
\end{aligned}
$$

Note that the vanishing of the tilting angle $v$ in the velocity vector also corroborates the conformal flatness of the spacetime geometry for $k=0$.

5.2. The Case $k>0$. The energy density, pressure, and soundspeed parameter have the forms

$$
\begin{gathered}
\mu=-3 k \widetilde{g}^{2}+3 \alpha\left(\frac{-\alpha}{2 k}+g\right)+4 k\left(\frac{-\alpha}{2 k}+g\right)^{2}, \\
p=3 k \widetilde{g}^{2}-\left(\frac{-\alpha}{2 k}+g\right)(\alpha+2 k g), \\
\frac{d p}{d \mu}=1-\frac{\alpha}{2 k g},
\end{gathered}
$$

respectively, and we have made the redefinitions $g=$ $C_{1} \cosh \sqrt{k} x+C_{2} \sinh \sqrt{k} x$ and $\tilde{g}=C_{2} \cosh \sqrt{k} x+$ $C_{1} \sinh \sqrt{k} x$. In order to satisfy the weak, strong, and dominant energy conditions, we require that each of

$$
\begin{gathered}
\mu-p=-6 k \tilde{g}^{2}+2\left(\frac{-\alpha}{2 k}+g\right)(\alpha+4 g), \\
\mu+p=2 k g\left(\frac{-\alpha}{2 k}+g\right), \\
\mu+3 p=6 k \tilde{g}^{2}+\frac{(\alpha+k g)(\alpha-2 k g)}{k}
\end{gathered}
$$

be positive.

Now, it remains to select suitable values of the four constants in the problem in order to generate an astrophysical model that satisfies elementary conditions for physical reality. In order to reduce the arbitrariness in selecting these constants, we may consider the behaviour of the dynamical variables at the central axis corresponding to $x=0$-with the idea being that the constants should be compatible with 
a regular centre in the first place. Using the subscript 0 to denote central values, we obtain

$$
\begin{gathered}
\rho_{0}=\frac{6 k^{2} C_{2}^{2}-8 k^{2} C_{1}^{2}+2 \alpha k C_{1}+\alpha^{2}}{k} \leq 0, \\
p_{0}=\frac{6 k^{2} C_{2}^{2}-4 k^{2} C_{1}^{2}-\alpha^{2}}{k} \geq 0, \\
0<\left(\frac{d p}{d \rho}\right)_{0}=1-\frac{\alpha}{2 k C_{1}}<1,
\end{gathered}
$$

where it must be recalled that $\alpha$ and $k$ are positive. The rightmost inequality of (64) implies that $C_{1}>0$, and the left inequality requires that $C_{1}>\alpha / 2 k$. In addition, (62) and (63) lead to $\alpha^{2}+4 k^{2} C_{1}^{2} \leq 6 k^{2} C_{2}^{2} \leq 8 k^{2} C_{1}^{2}+2 \alpha k C_{1}+\alpha^{2}$, which places a constraint on $C_{2}$. Empirical testing with these constraints in mind results in the prescription $k=1, \alpha=2$, $C_{1}=12 / 5$, and $C_{2}=-2$ which produces pleasing physical properties. Graphical representations of the dynamical and energy profiles are shown in Figures 1 and 2, respectively.

From Figure 1, we observe that both the energy and pressure are positive definite and monotonically decreasing functions of the space variable $x$. Importantly, there exists a pressure-free hypersurface at $x=0,11458$ which identifies a boundary for the distribution of the perfect fluid. Therefore, this model may succeed in modeling astrophysical phenomena. In addition, it can be seen that the adiabatic sound-speed index $d p / d \mu$ remains constrained between 0 and 1 throughout the interior of this fluid configuration. This is an indication that the sound speed is subluminal; that is, the sound speed never exceeds the speed of light, which is a fundamental postulate of the Einstein theory of the gravitational field. Figure 2 reveals that the weak, strong, and dominant energy conditions $(\mu-p>0, \mu+p>0$ and $\mu+$ $3 p>0$, resp.) are all satisfied throughout the fluid.

5.3. The Case $k<0$. In this case, the energy density, pressure and sound-speed index are given by

$$
\begin{gathered}
\mu=3 k \widetilde{j}^{2}+\left(\frac{-\alpha}{2 k}+j\right)(\alpha+4 k j), \\
p=-3 k \widetilde{j}^{2}-\left(\frac{-\alpha}{2 k}+j\right)(\alpha+2 k j), \\
\frac{d p}{d \mu}=1-\frac{\alpha}{2 k j}
\end{gathered}
$$

where we have defined $j=C_{1} \cos \sqrt{-k} x+C_{2} \sin \sqrt{-k} x$ and $\tilde{j}=C_{2} \cos \sqrt{-k} x-C_{1} \sqrt{-k} x$. The quantities

$$
\begin{gathered}
\mu-p=6 k \tilde{j}^{2}+\frac{(-\alpha+2 k j)(\alpha+3 k j)}{k}, \\
\mu+p=\frac{k j(-\alpha+k j)}{k}, \\
\mu+3 p=-6 k \widetilde{j}^{2}-\frac{(-\alpha+2 k j)(\alpha+k j)}{k}
\end{gathered}
$$

will assist in studying the energy conditions.

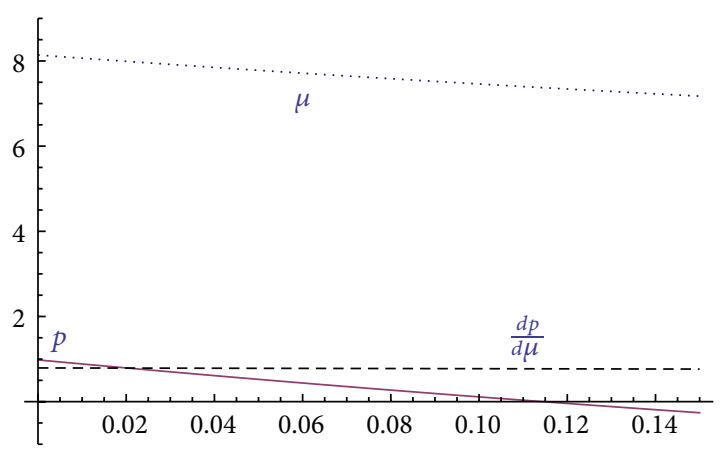

FIGURE 1: Graph of energy density $(\mu)$, pressure $(p)$ and sound-speed index $(d p / d \mu)$.

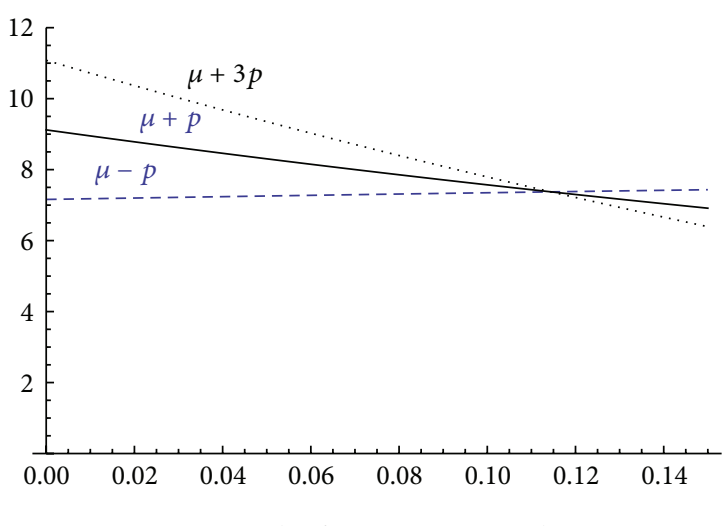

FIGURE 2: Graph of $\mu-p, \mu+p$, and $\mu+3 p$.

As above we now need to find suitable constants in order to create a viable fluid model. Again we examine the above quantities at the central axis $x=0$. We obtain

$$
\begin{gathered}
\rho_{0}=\frac{6 k^{2} C_{2}^{2}+\left(-\alpha+2 k C_{1}\right)\left(\alpha+4 k C_{1}\right)}{2 k}>0, \\
p_{0}=\frac{-6 k^{2} C_{2}^{2}-\left(-\alpha+2 k C_{1}\right)\left(\alpha+2 k C_{1}\right)}{2 k} \geq 0, \\
0 \leq \frac{d p}{d \mu}=1-\frac{\alpha}{2 k C_{1}}<1
\end{gathered}
$$

as constraints on the constants. Now, from (71), we infer that $r<0$ and also that $\alpha<2 k C_{1}$ recalling that $\alpha>0$ and $k<0$ in this case. Furthermore, reconciling (69) and (70), we obtain the relations $\alpha^{2}-4 k^{2} C_{1}^{2}<6 k^{2} C_{2}^{2}<\alpha^{2}+2 \alpha k C_{1}-8 k^{2} C_{1}^{2}$. Disregarding the middle term in this inequality, we derive that $\alpha>2 k C_{1}$ which contradicts our earlier conclusion. Therefore, it is impossible to satisfy the three most important physical requirements $\rho>0, p \geq 0$, and $0<d p / d \mu<1$ with any combination of the constants in the problem. In other words, the case $k<0$ cannot represent a realistic fluid. Therefore, we do not pursue any further consideration of this case. 


\section{Nonstatic Solutions}

Finding nonstatic exact solutions of the Einstein equations for a particular configuration of matter is nontrivial. The method of conformal transformations gives us the ability to construct non-static solutions from known static metrics. In the case under investigation, we suppose that the conformal factor has the simple form $U=U(t)$. The field equations then have the familiar form

$$
\begin{gathered}
\mu=3 f_{t}^{2}+f(4 k f+3 \alpha), \\
p=-3 f_{t}^{2}+f\left(2 f_{t t}-\alpha\right), \\
\tanh v=\frac{-(2 k f+\alpha)}{2 f_{t t}+2 k f+\alpha}, \\
0=(2 k f+\alpha)\left(2 f_{t t}+2 k f+\alpha\right),
\end{gathered}
$$

where now $f=f(t)$ only. Once again, the conclusion $f(t)=\alpha / 2 k$ leads to a trivial constant conformal factor, so we disregard it. We examine the other possibility

$$
2 f_{t t}+2 k f+\alpha=0
$$

more closely. Equation (73) is a simple second-order differential equation and is solved by

$$
f(x)= \begin{cases}-\frac{\alpha}{4} t^{2}+C_{1} t+C_{2}, & k=0, \\ -\frac{\alpha}{2 k}+C_{1} \cos \sqrt{k} t+C_{2} \sin \sqrt{k} t, & k>0, \\ \frac{\alpha}{2 k}+C_{1} \cosh \sqrt{-k} t+C_{2} \sinh \sqrt{-k} t, & k<0\end{cases}
$$

where $C_{1}$ and $C_{2}$ are integration constants. Let us examine each case in turn.

6.1. The Case $k=0$. This case degenerates into a conformal flat metric and so it is not worthy of further attention as such metrics have already been discovered.

6.2. The Case $k>0$. If we make the change of variables $l=$ $C_{1} \cos \sqrt{k} t+C_{2} \sin \sqrt{k} t$, and $\tilde{l}=C_{2} \cos \sqrt{k} t-C_{1} \sin \sqrt{k} t$ then the relevant dynamical quantities have the following forms:

$$
\begin{gathered}
\mu=-3 k \tilde{l}^{2}-\frac{1}{2 k}(\alpha-2 k l)(\alpha+4 k l), \\
p=3 k \tilde{l}^{2}+\frac{1}{2 k}(\alpha-2 k l)^{2}, \\
\frac{d p}{d \mu}=\frac{\alpha-14 k l}{2(\alpha+k l)} .
\end{gathered}
$$

It is pleasing to note that the positive definiteness of pressure criterion is valid everywhere for this case $k>0$. We also expect all physical constraints to be satisfied at the initial condition $t=0$. Denoting initial values with the subscript 0 , we have that the following inequalities must be satisfied:

$$
\begin{gathered}
\mu_{0}=\frac{-6 k^{2} C_{2}^{2}-\alpha^{2}-2 \alpha k C_{1}+8 k^{2} C_{1}^{2}}{2 k}>0, \\
p_{0}=\frac{6 k^{2} C_{2}^{2}+\alpha^{2}-4 \alpha k C_{1}+4 k^{2} C_{1}^{2}}{2 k} \geq 0, \\
0<\left(\frac{d p}{d \mu}\right)_{0}=\frac{\alpha-14 k C_{1}}{2\left(\alpha+k C_{1}\right)}<1 .
\end{gathered}
$$

The first two relationships constrain $k$ as

$$
\begin{aligned}
-\alpha^{2} & +4 \alpha k C_{1}-4 k^{2} C_{1}^{2} \\
& \leq 6 k^{2} C_{2}^{2}<-\alpha^{2}-2 \alpha k C_{1}+8 k^{2} C_{1}^{2},
\end{aligned}
$$

and the outer two expressions must satisfy $C_{1}<0$ or $C_{1}>$ $\alpha / 2 k$. The adiabatic sound-speed criterion (78) forces the value of $C_{1}$ as $-\alpha / 16 k<C_{1}<\alpha / 14 k$ since $\alpha>0$ and $k>0$. An additional constraint on $C_{1}$ is implied by requiring the positivity of the right hand side of (79). This is satisfied provided that $C_{1}<-\alpha / 4 k$ or $C_{1}>\alpha / 2 k$. Now, it can immediately be seen that these inequalities are never satisfied simultaneously for any value of $C_{1}$. Accordingly, we deduce that no physical reasonable model will result from this case. That is, positivity of pressure and energy density as well as a subluminal sound speed cannot all be achieved for any value of the constant $C_{1}$. There is little value in considering the value of $C_{2}$ in view of the nonsatisfaction of these requirements.

6.3. The Case $k<0$. In this case, the energy density, pressure, and sound-speed index are given, respectively, by

$$
\begin{gathered}
\mu=\frac{6 k * \widetilde{v}^{2}+5 \alpha^{2}+14 \alpha k v+8 k^{2} v^{2}}{2 k}, \\
p=\frac{-6 k \widetilde{v}^{2}-\alpha^{2}+4 k^{2} v^{2}}{2 k}, \\
\frac{d p}{d \mu}=-7\left(\frac{\alpha}{2 k v}+1\right),
\end{gathered}
$$

where we have again redefined $v=C_{1} \cosh \sqrt{-k} t+$ $C_{2} \sinh \sqrt{-k} t$ and $\widetilde{v} C_{2} \cosh \sqrt{-k} t+C_{1} \sinh \sqrt{-k} t$. Reasoning as before together with empirical testing corroborates that it is not possible to achieve simultaneously a positive pressure and energy density. Additionally, the sound-speed index is always found to be negative. Therefore, this model cannot model a realistic distribution of stellar fluid.

\section{A Spacetime Foliation $U=U(t, x)$}

The Lie group analysis method has proved successful in generating broader classes of solutions than the ones discussed previously. The functional forms for $f(t, x)$ for $h=0$ were previously obtained by Hansraj et al. [27] using group analysis techniques. We now take these solutions and substitute into expressions (47) and (48) to establish the dynamical 
quantities, energy density $(\mu)$ and pressure $(p)$. Thereafter, we verify that the pressure and density profiles satisfy conditions for physical acceptability. A rigorous analytical treatment is prohibitive for general $f(t, x)$ and $h(y, z)$, in view of the complexity of the resultant expressions. Therefore, for graphical purposes, it is necessary to consider a simplified situation such as foliations of the distribution in terms of the temporal and one space variable. It is not possible to visually represent the complete 4-dimensional solution spacetime. We accordingly select the particular solution

$$
f(x, t)=A \sinh \sqrt{k\left(t^{2}-x^{2}\right)}+B \cosh \sqrt{k\left(t^{2}-x^{2}\right)}
$$

presented by Hansraj et al. [27] together with that obtained from $Z_{1}$. The conformally related metric (33) may now be given by

$$
\begin{aligned}
d s^{2}= & \left(-d t^{2}+d x^{2}+c_{1} e^{z^{2} / 8 y^{2}}\left(\frac{z}{y}\right)^{3 / 4}\right. \\
& \left.\times\left(\frac{z^{2}}{y^{2}}-1\right)^{1 / 2}\left(d y^{2}+d z^{2}\right)\right) \\
& \times\left(\left(e^{c_{1}+z^{2} / 8 y^{2}} y^{3 / 4} z^{1 / 4}+B \cosh \left[\sqrt{k\left(t^{2}-x^{2}\right)}\right]\right.\right. \\
& \left.\left.\quad+A \sinh \left[\sqrt{k\left(t^{2}-x^{2}\right)}\right]\right)^{2}\right)^{-1},
\end{aligned}
$$

and the energy density takes the form

$$
\begin{aligned}
\mu= & -3\left(c_{1} e^{z^{2} / 8 y^{2}}\left(\frac{z}{y}\right)^{3 / 4}\left(\frac{z^{2}}{y^{2}}-1\right)^{1 / 2}\right)^{-1} \\
& \times\left(\left(\frac{e^{c_{1}+z^{2} / 8 y^{2}} y^{3 / 4}}{4 z^{3 / 4}}+\frac{e^{c_{1}+z^{2} / 8 y^{2}} z^{5 / 4}}{4 y^{5 / 4}}\right)^{2}\right. \\
& +\left(\frac{3 e^{c_{1}+z^{2} / 8 y^{2}} z^{1 / 4}}{\left.\left.4 y^{1 / 4}-\frac{e^{c_{1}+z^{2} / 8 y^{2}} z^{9 / 4}}{4 y^{9 / 4}}\right)^{2}\right)}\right) \\
& \left(e^{c_{1}+z^{2} / 8 y^{2}} y^{3 / 4} z^{1 / 4}+B \cosh \left[\sqrt{k\left(t^{2}-x^{2}\right)}\right]\right. \\
& \left.+A \sinh \left[\sqrt{k\left(t^{2}-x^{2}\right)}\right]\right) \\
& +2 e^{c_{1}+z^{2} / 8 y^{2}} k y^{3 / 4} z^{1 / 4}-3 \alpha \\
& +4 k\left(B \cosh \left[\sqrt{k\left(t^{2}-x^{2}\right)}\right]\right. \\
+ & \left.\left.+A \sinh \left[\sqrt{k\left(t^{2}-x^{2}\right)}\right]\right)\right)
\end{aligned}
$$

$$
\begin{gathered}
+3\left(\left(\frac{A k t \cosh \left[\sqrt{k\left(t^{2}-x^{2}\right)}\right]}{\sqrt{k\left(t^{2}-x^{2}\right)}}\right)\right. \\
\left.+\frac{B k t \sinh \left[\sqrt{k\left(t^{2}-x^{2}\right)}\right]}{\sqrt{k\left(t^{2}-x^{2}\right)}}\right)^{2} \\
-\left(\frac{A k x \cosh \left[\sqrt{k\left(t^{2}-x^{2}\right)}\right]}{\sqrt{k\left(t^{2}-x^{2}\right)}}\right. \\
\left.\left.-\frac{B k x \sinh \left[\sqrt{k\left(t^{2}-x^{2}\right)}\right]}{\sqrt{k\left(t^{2}-x^{2}\right)}}\right)^{2}\right),
\end{gathered}
$$

while the pressure is given by

$$
\begin{aligned}
& p=3\left(c_{1} e^{z^{2} / 8 y^{2}}\left(\frac{z}{y}\right)^{3 / 4}\left(\frac{z^{2}}{y^{2}}-1\right)^{1 / 2}\right)^{-1} \\
& \times\left(\left(\frac{e^{c_{1}+z^{2} / 8 y^{2}} y^{3 / 4}}{4 z^{3 / 4}}+\frac{e^{c_{1}+z^{2} / 8 y^{2}} z^{5 / 4}}{4 y^{5 / 4}}\right)^{2}\right. \\
& \left.+\left(\frac{3 e^{c_{1}+z^{2} / 8 y^{2}} z^{1 / 4}}{4 y^{1 / 4}}-\frac{e^{c_{1}+z^{2} / 8 y^{2}} z^{9 / 4}}{4 y^{9 / 4}}\right)^{2}\right) \\
& -\frac{k\left(3 t^{2}+x^{2}\right)}{\left(t^{2}-x^{2}\right)}\left(A \cosh \left[\sqrt{k\left(t^{2}-x^{2}\right)}\right]\right. \\
& \left.+B \sinh \left[\sqrt{k\left(t^{2}-x^{2}\right)}\right]\right)^{2} \\
& +\left(e^{c_{1}+z^{2} / 8 y^{2}} y^{3 / 4} z^{1 / 4}+B \cosh \left[\sqrt{k\left(t^{2}-x^{2}\right)}\right]\right. \\
& \left.+A \sinh \left[\sqrt{k\left(t^{2}-x^{2}\right)}\right]\right) \\
& \times(-\alpha+2 \\
& \times\left(e^{c_{1}+z^{2} / 8 y^{2}} k y^{3 / 4} z^{1 / 4}\right. \\
& +A\left(-\frac{k^{2} t^{2} \cosh \left[\sqrt{k\left(t^{2}-x^{2}\right)}\right]}{\left(k\left(t^{2}-x^{2}\right)\right)^{3 / 2}}\right.
\end{aligned}
$$




$$
\begin{aligned}
& +\frac{k \cosh \left[\sqrt{k\left(t^{2}-x^{2}\right)}\right]}{\sqrt{k\left(t^{2}-x^{2}\right)}} \\
& \left.+\frac{k t^{2} \sinh \left[\sqrt{k\left(t^{2}-x^{2}\right)}\right]}{t^{2}-x^{2}}\right) \\
& -A\left(-\frac{k^{2} x^{2} \cosh \left[\sqrt{k\left(t^{2}-x^{2}\right)}\right]}{\left(k\left(t^{2}-x^{2}\right)\right)^{3 / 2}}\right. \\
& -\frac{k \cosh \left[\sqrt{k\left(t^{2}-x^{2}\right)}\right]}{\sqrt{k\left(t^{2}-x^{2}\right)}} \\
& \left.+\frac{k x^{2} \sinh \left[\sqrt{k\left(t^{2}-x^{2}\right)}\right]}{t^{2}-x^{2}}\right) \\
& -B\left(\frac{k x^{2} \cosh \left[\sqrt{k\left(t^{2}-x^{2}\right)}\right]}{t^{2}-x^{2}}\right. \\
& -\frac{k^{2} x^{2} \sinh \left[\sqrt{k\left(t^{2}-x^{2}\right)}\right]}{\left(k\left(t^{2}-x^{2}\right)\right)^{3 / 2}} \\
& \left.-\frac{k \sinh \left[\sqrt{k\left(t^{2}-x^{2}\right)}\right]}{\sqrt{k\left(t^{2}-x^{2}\right)}}\right) \\
& +B\left(-\frac{k t^{2} \cosh \left[\sqrt{k\left(t^{2}-x^{2}\right)}\right]}{t^{2}-x^{2}}\right. \\
& -\frac{k^{2} t^{2} \sinh \left[\sqrt{k\left(t^{2}-x^{2}\right)}\right]}{\left(k\left(t^{2}-x^{2}\right)\right)^{3 / 2}} \\
& \left.\left.+\frac{k \sinh \left[\sqrt{k\left(t^{2}-x^{2}\right)}\right]}{\sqrt{k\left(t^{2}-x^{2}\right)}}\right)\right) \text {. }
\end{aligned}
$$

In order to obtain an indication of the model's feasibility to represent a realistic distribution, we elect to make a graphical study of this solution. The energy density and pressure are given by

$$
\begin{aligned}
\mu=4 k\left(B \cosh \left[\sqrt{k\left(t^{2}-x^{2}\right)}\right]\right. \\
\left.+A \sinh \left[\sqrt{k\left(t^{2}-x^{2}\right)}\right]\right)^{2}
\end{aligned}
$$$$
-3\left(\left(\frac{A k t \cosh \left[\sqrt{k\left(t^{2}-x^{2}\right)}\right]}{\sqrt{k\left(t^{2}-x^{2}\right)}}\right.\right.
$$$$
\left.+\frac{B k t \sinh \left[\sqrt{k\left(t^{2}-x^{2}\right)}\right]}{\sqrt{k\left(t^{2}-x^{2}\right)}}\right)^{2}
$$$$
-\left(\frac{A k x \cosh \left[\sqrt{k\left(t^{2}-x^{2}\right)}\right]}{\sqrt{k\left(t^{2}-x^{2}\right)}}\right.
$$$$
\left.\left.-\frac{B k x \sinh \left[\sqrt{k\left(t^{2}-x^{2}\right)}\right]}{\sqrt{k\left(t^{2}-x^{2}\right)}}\right)^{2}\right) \text {, }
$$

$$
p=2\left(B \cosh \left[\sqrt{k\left(t^{2}-x^{2}\right)}\right]+A \sinh \left[\sqrt{k\left(t^{2}-x^{2}\right)}\right]\right)
$$$$
\times\left(A \left(-\frac{k^{2} t^{2} \cosh \left[\sqrt{k\left(t^{2}-x^{2}\right)}\right]}{\left(k\left(t^{2}-x^{2}\right)\right)^{3 / 2}}\right.\right.
$$$$
+\frac{k \cosh \left[\sqrt{k\left(t^{2}-x^{2}\right)}\right]}{\sqrt{k\left(t^{2}-x^{2}\right)}}
$$$$
\left.+\frac{k t^{2} \sinh \left[\sqrt{k\left(t^{2}-x^{2}\right)}\right]}{t^{2}-x^{2}}\right)
$$$$
-A\left(\frac{-k^{2} x^{2} \cosh \left[\sqrt{k\left(t^{2}-x^{2}\right)}\right]}{\left(k\left(t^{2}-x^{2}\right)\right)^{3 / 2}}\right.
$$$$
-\frac{k \cosh \left[\sqrt{k\left(t^{2}-x^{2}\right)}\right]}{\sqrt{k\left(t^{2}-x^{2}\right)}}
$$$$
\left.+\frac{k x^{2} \sinh \left[\sqrt{k\left(t^{2}-x^{2}\right)}\right]}{t^{2}-x^{2}}\right)
$$

$$
\begin{array}{r}
-B\left(\frac{k x^{2} \cosh \left[\sqrt{k\left(t^{2}-x^{2}\right)}\right]}{t^{2}-x^{2}}\right. \\
-\frac{k^{2} x^{2} \sinh \left[\sqrt{k\left(t^{2}-x^{2}\right)}\right]}{\left(k\left(t^{2}-x^{2}\right)\right)^{3 / 2}} \\
\left.-\frac{k \sinh \left[\sqrt{k\left(t^{2}-x^{2}\right)}\right]}{\sqrt{k\left(t^{2}-x^{2}\right)}}\right)
\end{array}
$$




$$
\begin{gathered}
+B\left(\frac{k t^{2} \cosh \left[\sqrt{k\left(t^{2}-x^{2}\right)}\right]}{t^{2}-x^{2}}\right. \\
-\frac{k^{2} t^{2} \sinh \left[\sqrt{k\left(t^{2}-x^{2}\right)}\right]}{\left(k\left(t^{2}-x^{2}\right)\right)^{3 / 2}} \\
\left.\left.+\frac{k \sinh \left[\sqrt{k\left(t^{2}-x^{2}\right)}\right]}{\sqrt{k\left(t^{2}-x^{2}\right)}}\right)\right) \\
-\frac{k\left(3 t^{2}+x^{2}\right)}{\left(t^{2}-x^{2}\right)} \\
\times\left(A \cosh \left[\sqrt{k\left(t^{2}-x^{2}\right)}\right]\right. \\
\left.+B \sinh \left[\sqrt{k\left(t^{2}-x^{2}\right)}\right]\right)^{2}
\end{gathered}
$$

We need to select appropriate values for the parameters $A, B$, and $k$ to finalise the model. It should be observed at this stage that, in many situations, it is possible to obtain bounds for the constants by examining the central conditions $p(t, 0,0,0)$ and $\mu(t, 0,0,0)$. We then require that all the physical conditions are satisfied at the centre. This is not fruitful in the present context. Furthermore, the approach in fixing integration constants in most problems in general relativity involves matching of the perfect fluid solution with its corresponding vacuum solution. For example, if we were devising a model of a static neutral sphere, then integration constants can be fixed by demanding continuity of the potentials at the boundary hypersurface. In addition, for such solutions, the pressure vanishes at the boundary, and this determines the size of the sphere. In the case of radiating spheres, this pressure-free interface does not exist, and this condition is modified to include the effects of radiation, and the junction conditions must be established via other means. The difficulty in our analysis is that we have no known vacuum solution. Consequently, our selection of the constants is completely random. We are fortunate that the available computing technology is able to quickly check the efficacy of our choices for the integration constants (see Figures 3 and 4).

These plots, generated via Mathematica [35], demonstrate many pleasing features. For example, it is evident that, in the region chosen, the pressure and energy density are both positive. These are the most basic requirements for models to serve as candidates for realistic celestial phenomena. Therefore, it is possible for this solution to model a realistic configuration of perfect fluid.

\section{Conclusion}

We have demonstrated that new static and nonstatic solutions of the Einstein field equations may be constructed from

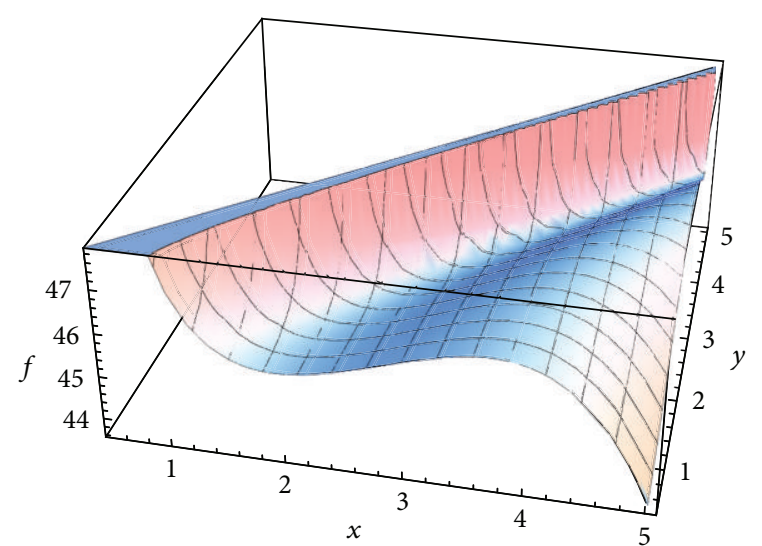

Figure 3: Plot of pressure versus $t$ and $x$ with $A=1, B=10$, and $k=0.1$.

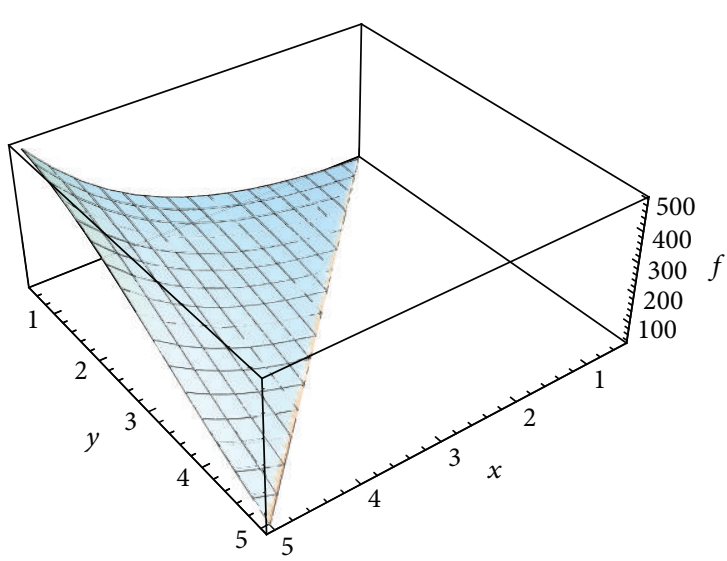

Figure 4: Plot of energy density versus $t$ and $x$ with $A=1, B=$ 10 , and $k=0.1$.

known solutions through the use of conformal mappings. Even solutions that are considered as defective may serve as seeds for new solutions which are physically reasonable. The reason for the success of this approach is that the seed solution is already a solution of the possibly vacuum Einstein field equations. The Einstein tensor splits up neatly into a part containing the original Einstein tensor and an expression involving the conformal factor. It is this latter part that must be coupled to an appropriate perfect fluid. The mathematical reason for this behaviour is that the conformal Killing vectors of the original seed metric become Killing vectors of the new metric. This generates symmetries which have the effect of simplifying the resultant field equations. While 4-dimensional solutions to the field equations have already been reported, solutions had not been analysed for physical plausibility. We have endeavoured to construct explicit models by considering cases where the conformal factor is a function of a space variable $U=U(x)$, a function of the temporal coordinate $U=U(t)$ only and finally the case of the conformal factor as a function of space and time $U=U(t, x)$. Analyses of each case show that it is possible to 
generate physically reasonable models through this method of conformal transformations.

\section{References}

[1] G. D. Birkhoff, Relativity and Modern Physics, Harvard University Press, Cambridge, Mass, USA, 1923.

[2] K. Schwarzschild, "Über das Gravitationsfeld eines Massenpunktes nach der Einsteinschen Theorie," Sitzungsberichte Der Preussischen Akademie Der Wissenschaften, PhysikalischMathematische, vol. K1, p. 189, 1916.

[3] K. Schwarzschild, "Über das Gravitationsfeld einer Kugel aus inkompressibler Flüssigkeit," Sitzungsberichte Der Preussischen Akademie Der Wissenschaften, Physikalisch-Mathematische, vol. K1, p. 424, 1916.

[4] M. S. R. Delgaty and K. Lake, "Physical acceptability of isolated, static, spherically symmetric, perfect fluid solutions of Einstein's equations," Computer Physics Communications, vol. 115, no. 2-3, pp. 395-415, 1998.

[5] G. Fodor, "Generating spherically symmetric static perfect fluid solutions," http://arxiv.org/abs/gr-qc/0011040.

[6] D. Martin and M. Visser, "Algorithmic construction of static perfect fluid spheres," Physical Review D, vol. 69, no. 10, Article ID 104028, 2004.

[7] K. Lake, "All static spherically symmetric perfect-fluid solutions of Einstein's equations," Physical Review D, vol. 67, no. 10, Article ID 104015, 2003.

[8] P. Boonserm, M. Visser, and S. Weinfurtner, "Generating perfect fluid spheres in general relativity," Physical Review D, vol. 71, no. 12, Article ID 124037, 2005.

[9] S. Rahman and M. Visser, "Spacetime geometry of static fluid spheres," Classical and Quantum Gravity, vol. 19, no. 5, pp. 935952, 2002.

[10] M. Wyman, "Radially symmetric distributions of matter," Physical Review, vol. 75, pp. 1930-1936, 1949.

[11] M. R. Finch and J. E. F. Skea, 1998, http://www.dft.if.uerj.br/ usuarios/JimSkea/papers/pfrev.ps.

[12] B. V. Ivanov, "Static charged perfect fluid spheres in general relativity," Physical Review D, vol. 65, no. 10, Article ID 104001, 2002.

[13] H. Reissner, "Über die Eigengravitation des elektrischen Feldes nach der Einsteinschen Theorie," Annales De Physique, vol. 50, p. 106, 1916.

[14] G. Nordstrom, "On the Energy of the Gravitational Field in Einstein's Theory," Proceedings of the Koninklijke Nederlandse Akademie Van Wetenschappen, Series B, vol. 20, Article ID 1238, 1918.

[15] S. Hansraj and S. D. Maharaj, "Charged analogue of Finch-Skea stars," International Journal of Modern Physics D, vol. 15, no. 8, pp. 1311-1327, 2006.

[16] P. C. Vaidya, "The gravitational field of a radiating star," Proceedings of the Indian Academy of Sciences A, vol. 33, pp. 264276, 1951.

[17] R. P. Kerr, "Gravitational field of a spinning mass as an example of algebraically special metrics," Physical Review Letters, vol. 11, pp. 237-238, 1963.

[18] H. L. Duorah and R. Ray, "An analytical stellar model," Classical and Quantum Gravity, vol. 4, no. 6, pp. 1691-1696, 1987.

[19] M. C. Durgapal and R. Bannerji, "New analytical stellar model in general relativity," Physical Review D, vol. 27, no. 2, pp. 328331,1983 .
[20] M. R. Finch and J. E. F. Skea, "A realistic stellar model based on an ansatz of Duorah and Ray," Classical and Quantum Gravity, vol. 6, no. 4, pp. 467-476, 1989.

[21] A. Z. Petrov, "Classification of spaces defined by gravitational fields," General Relativity and Gravitation, vol. 22, p. 1665, 1954.

[22] L. Defrise-Carter, "Conformal groups and conformally equivalent isometry groups," Communications in Mathematical Physics, vol. 40, pp. 273-282, 1975.

[23] G. S. Hall and J. D. Steele, "Conformal vector fields in general relativity," Journal of Mathematical Physics, vol. 32, no. 7, pp. 1847-1853, 1991.

[24] N. Van den Bergh, "Conformally Ricci-flat perfect fluids," Journal of Mathematical Physics, vol. 27, no. 4, pp. 1076-1081, 1986.

[25] N. van den Bergh, "Irrotational and conformally Ricci-flat perfect fluids," General Relativity and Gravitation, vol. 18, pp. 649-668, 1986.

[26] J. Castejón-Amenedo and A. A. Coley, "Exact solutions with conformal Killing vector fields," Classical and Quantum Gravity, vol. 9, no. 10, pp. 2203-2215, 1992.

[27] S. Hansraj, S. D. Maharaj, A. M. Msomi, and K. S. Govinder, "Lie symmetries for equations in conformal geometries," Journal of Physics A, vol. 38, no. 20, pp. 4419-4431, 2005.

[28] S. Hansraj, "Perfect fluid metrics conformal to the Schwarzschild exterior spacetime," General Relativity and Gravitation, vol. 44, no. 1, pp. 125-138, 2012.

[29] K. S. Govinder and S. Hansraj, "Group analysis of a conformal perfect fluid spacetime," Journal of Physics A, vol. 45, no. 15, Article ID 155210, 2012.

[30] G. H. Katzin and J. Levine, "Applications of Lie derivatives to symmetries, geodesic mappings, and first integrals in Riemannian spaces," Colloquium Mathematicum, vol. 26, pp. 21-38, 1972.

[31] G. H. Katzin, J. Levine, and W. R. Davis, "Curvature collineations: a fundamental symmetry property of the space-times of general relativity defined by the vanishing Lie derivative of the Riemann curvature tensor," Journal of Mathematical Physics, vol. 10, pp. 617-629, 1969.

[32] F. de Felice and C. J. S. Clarke, Relativity on Curved Manifolds, Cambridge Monographs on Mathematical Physics, Cambridge University Press, Cambridge, UK, 1990.

[33] B. O. J. Tupper, "Conformally Ricci-flat viscous fluids," Journal of Mathematical Physics, vol. 31, no. 7, pp. 1704-1706, 1990.

[34] H. Stephani, "Konform flache Gravitationsfelder," Communications in Mathematical Physics, vol. 5, no. 5, pp. 337-342, 1967.

[35] S. Wolfram, MATHEMATICA Version 8. 0, Addison-Wesley, Redwood City, Calif, USA, 2009. 


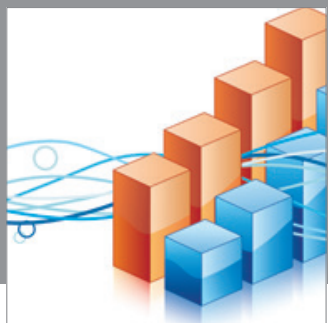

Advances in

Operations Research

mansans

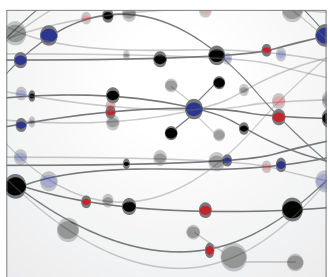

The Scientific World Journal
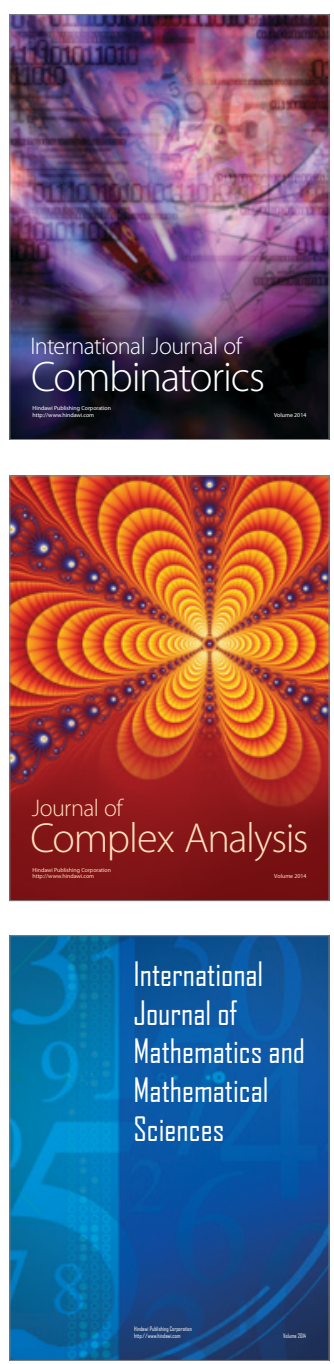
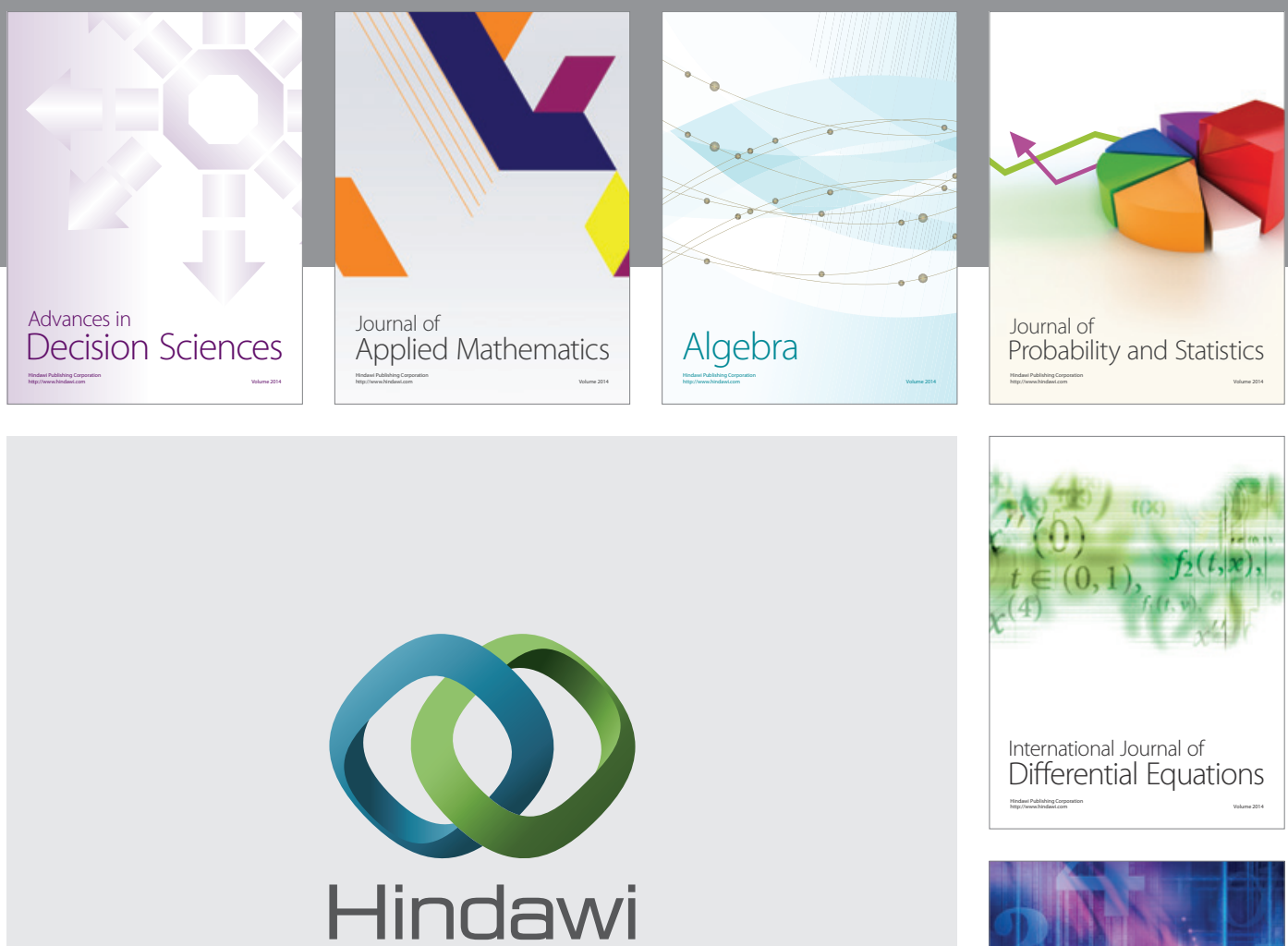

Submit your manuscripts at http://www.hindawi.com
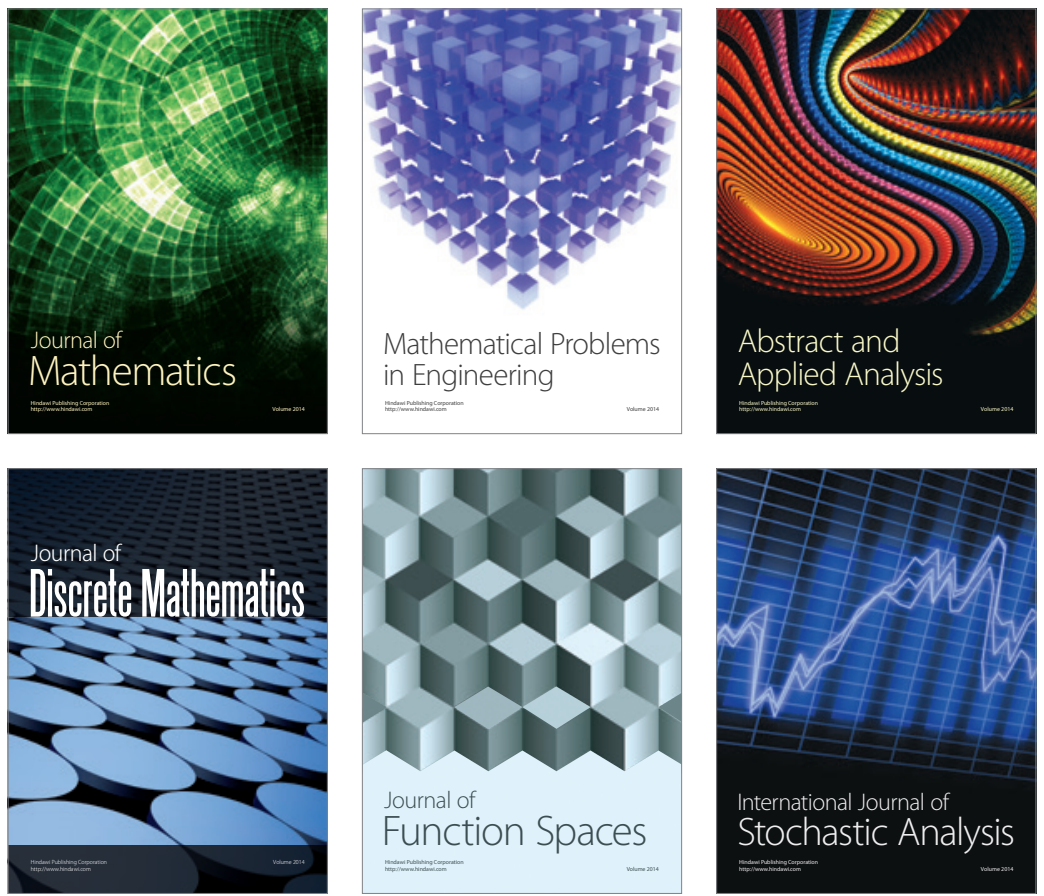

Journal of

Function Spaces

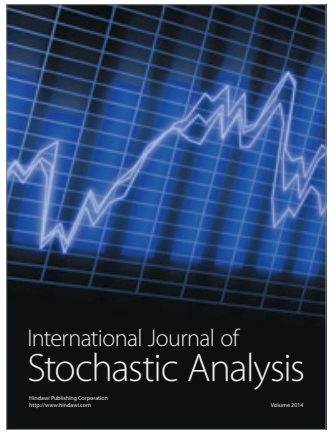

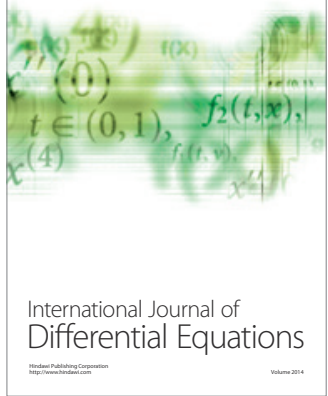
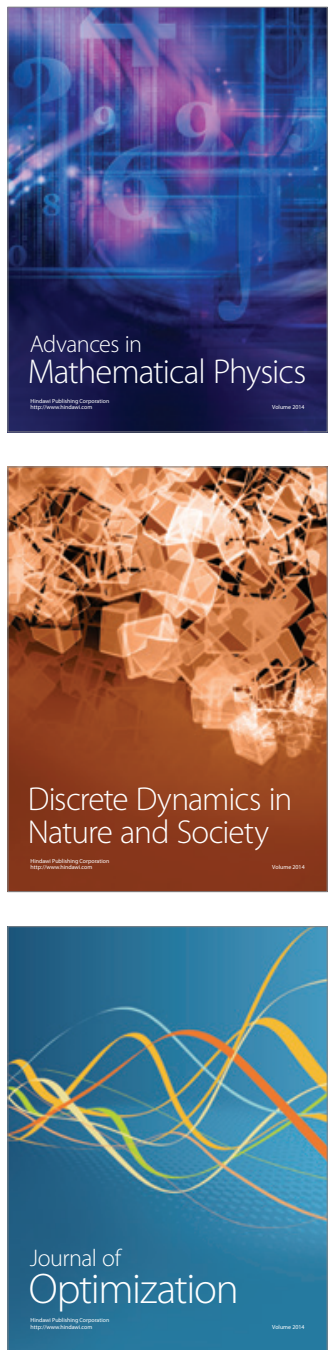